\title{
Orion Boiler Plate Airdrop Test System
}

\author{
Ricardo A. Machín ${ }^{1}$, Carol T. Evans ${ }^{2}$ \\ National Aeronautics and Space Administration, Johnson Space Center, Houston, TX 77058
}

On the 29th of February 2012 the Orion Capsule Parachute Assembly System (CPAS) project attempted to perform an airdrop test of a boilerplate test article for the second time. The first attempt (Cluster Development Test 2, July 2008) to deliver a similar boilerplate from a C-17 using the Low Velocity Air Drop (LVAD) technique resulted in the programmer parachute failing to properly inflate, the test article failing to achieve the desired test initiation conditions, and the test article a total loss. This paper will pick up where the CDT-2 failure investigation left off ${ }^{1}$, describing the test technique that was adopted, and outline the modeling that was performed to gain confidence that the second attempt would be successful. The second boiler plate test (Cluster Development Test 3-3) was indeed a complete success and has subsequently been repeated several times, allowing the CPAS project to proceed with the full scale system level development testing required to integrate the hardware to the first Entry Flight Test vehicle as well as go into the Critical Design Review with minimum risk and a mature design.

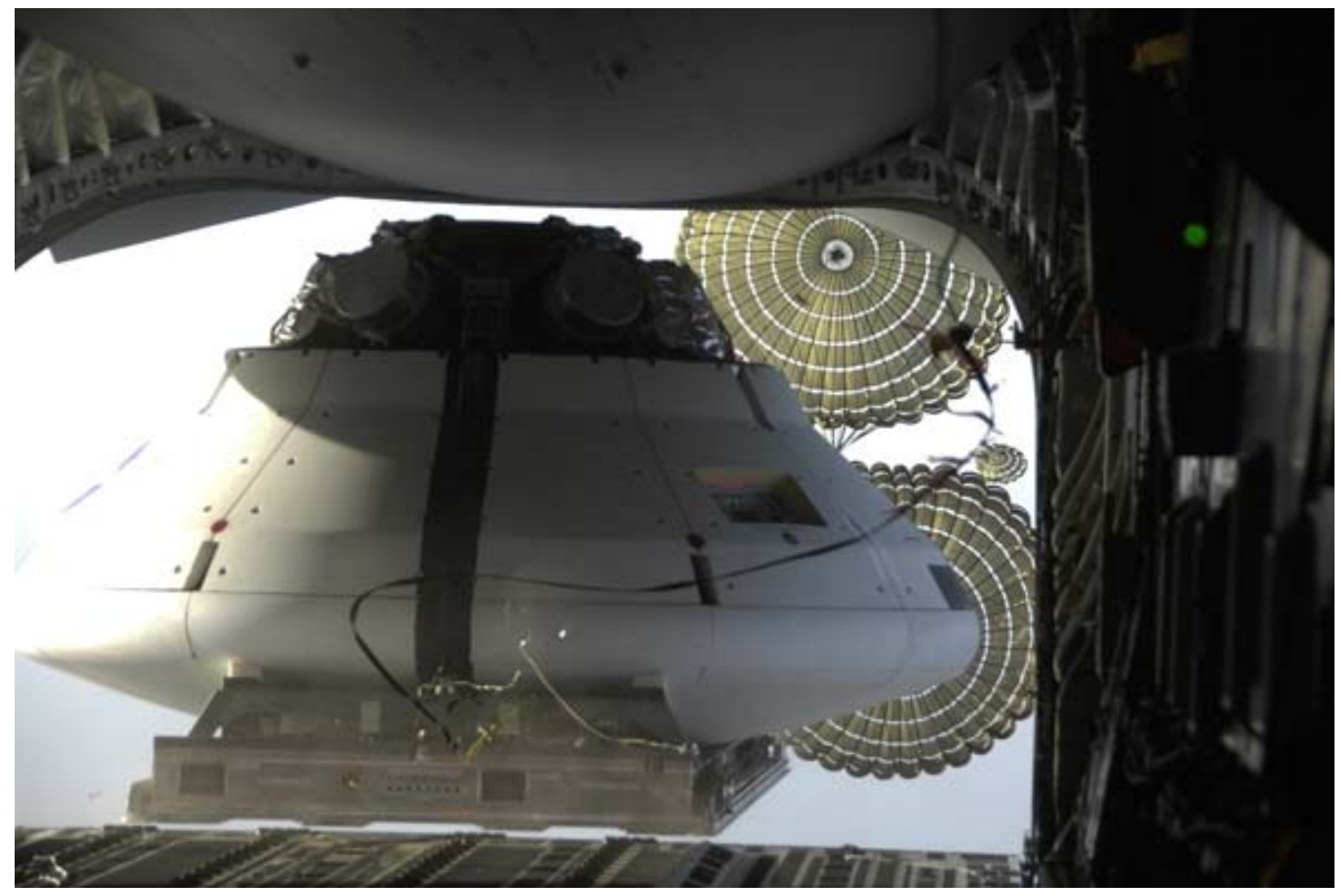

The Parachute Test Vehicle Immediately Following Extraction During CDT-3-3

\footnotetext{
${ }^{1}$ Orion CPAS Chief Engineer, Applied Aeroscience and CFD Branch, Mail Stop EG3, AIAA Member.

${ }^{2}$ Orion CPAS Test Lead, Test and Fabrication Branch, Mail Stop EA36.
} 


\section{Background (From One Test Vehicle to Two)}

The CDT-2 was the final planned test of the Gen-1 design ${ }^{2}$. Following this test, the project would be updating the Gen-1 design in order to hold the Preliminary Design Review (PDR) in August of 2010. The design coming out of the PDR was the Engineering Development Unit (EDU). As the CPAS Project advanced to the subsequent testing of the EDU parachute system the requirement for two distinctly different airdrop test vehicles was identified. Both test vehicles (figure 1) are built around a common full scale forward bay that replicates the Orion spacecraft. This forward bay provides the ability to implement the rigging and the retention system, and assess full scale deployment and performance of the CPAS design.
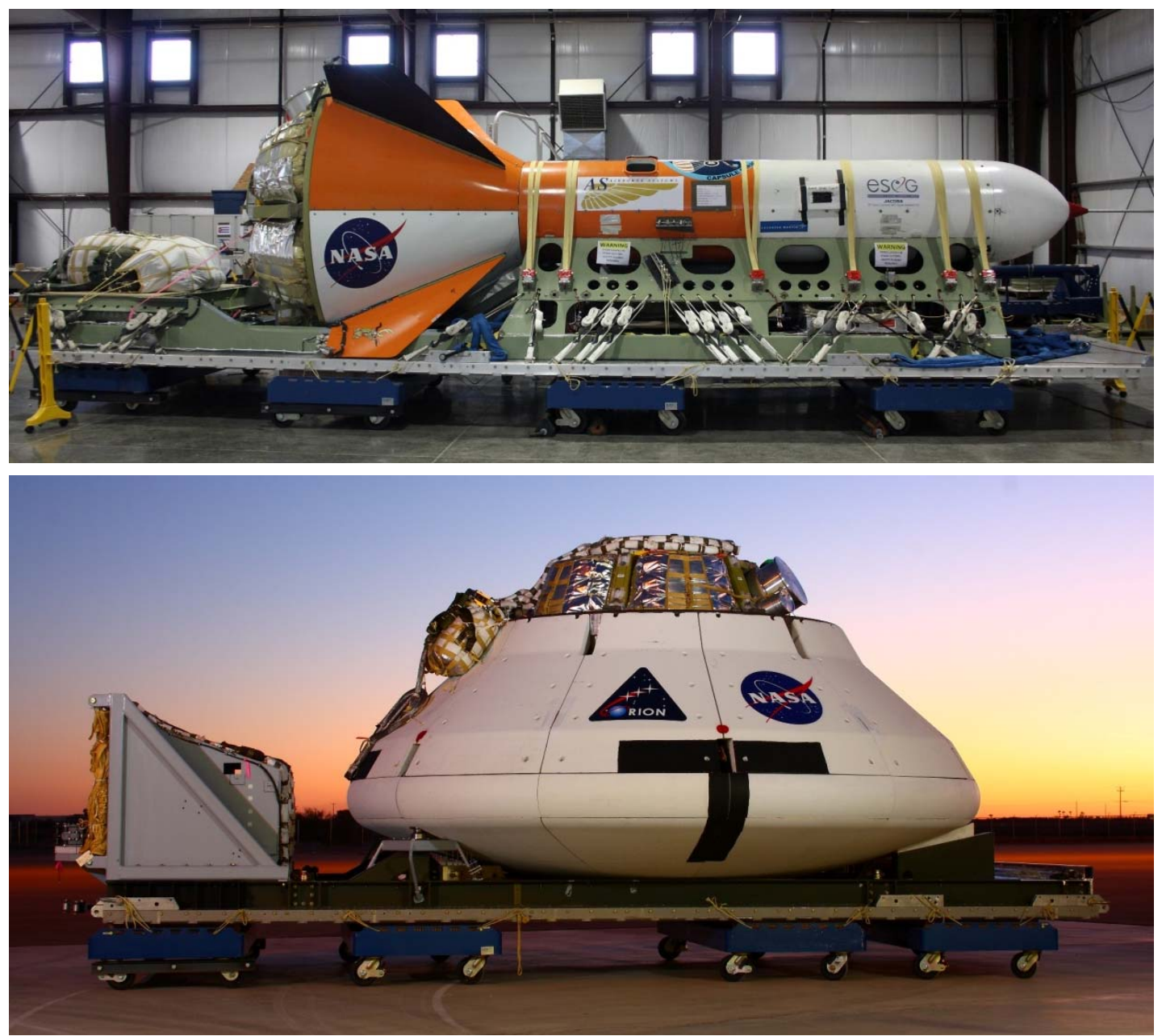

Figure 1. The PCDTV and the PTV Test Articles

The Parachute Compartment Drop Test Vehicle (PCDTV) is a missile shaped test article used to achieve higher dynamic pressure test conditions, it does not however subject the parachutes to the proper wake during testing. The PCDTV was designed to fit inside the extraction envelope for the United States Air Force C-130. The Parachute Test Vehicle (PTV) is a boiler plate with full a scale heat shield, but truncated in height in order to fit into the United States Air Force C-17 extraction envelope. The PTV is too large (height and width) to fit into a C-130, and will only be test from a C-17. The PTV does not have an active control system (required for controlled subsonic flight). As a result, the PTV cannot achieve the nominal entry deploy conditions using standard LVAD extraction altitudes as a starting point ${ }^{3}$. This limitation is most relevant to the Forward Bay Cover jettison and drogue phases of the CPAS design without loss of control. It does however provide the proper vehicle wake, allowing for the parachute models that are affected by the wake, as well as the models that predict the coupled dynamics of the drogues and capsule, to be anchored to test data.

There are a number of other design and architecture features the two test articles share. Both are attached to the platform with a metallic cradle and a textile restraint, referred to as the mated system. Each mated system has 
an extracted weight of between 31,000 and 32,000 lbs. For the PCDTV the platform (with its cradle) is called the Mid-air Delivery System(MDS). For the PTV this is referred to as the Carriage Platform Sled System (CPSS). Both test vehicles utilize the LVAD test technique to extract the mated vehicle from the delivery aircraft (C-130 or C-17). Both techniques implement a delayed load transfer to create an under-rotation that will result in an upswing of the mated system at the time the test vehicle is released from the platform. The textile restraint is pyrotechnically severed, initiated by a command from the mated vehicle sequencer. Both test vehicles transition to a programmer parachute with a multipoint attach harness, which is static line deployed by the platform (MDS or CPSS, depending on the test vehicle) as they separate. Figures 2 and 3 show the conceptual test sequence for the PCDTV and the PTV (respectively).

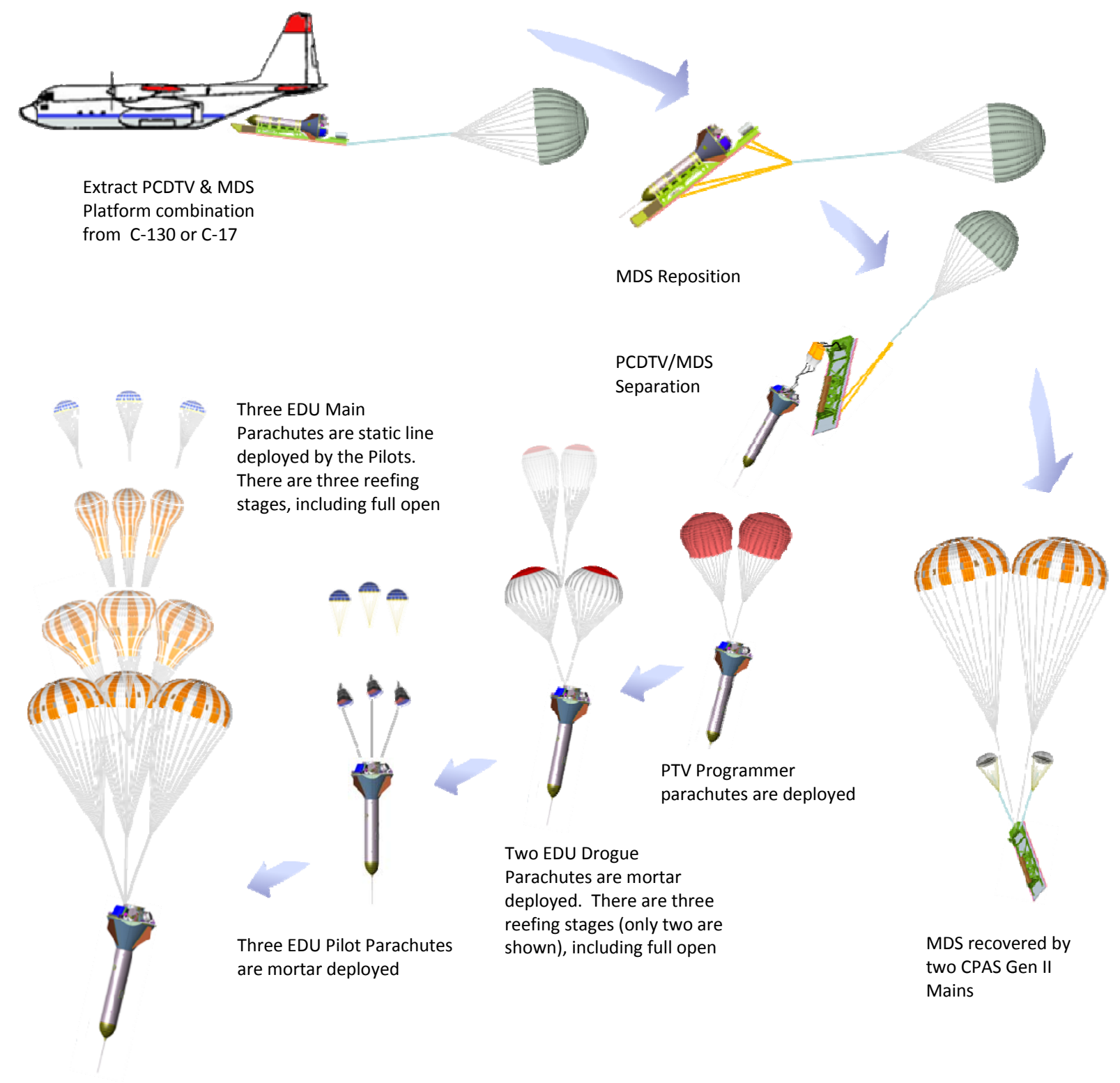

Figure 2. The PCDTV Test Sequence (including the MDS recovery) 


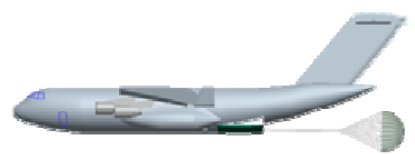

C-17 with a single $15 \mathrm{ft} \mathrm{GFE} \mathrm{Drogue}$ parachute prior to extraction

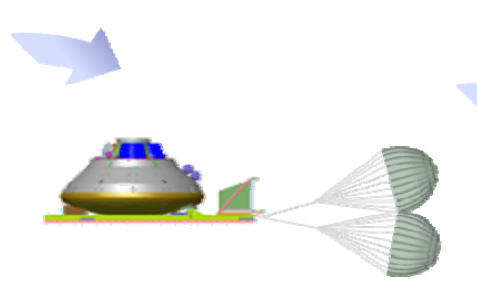

Extraction from C-17 with two $28 \mathrm{ft}$ Extraction Parachutes

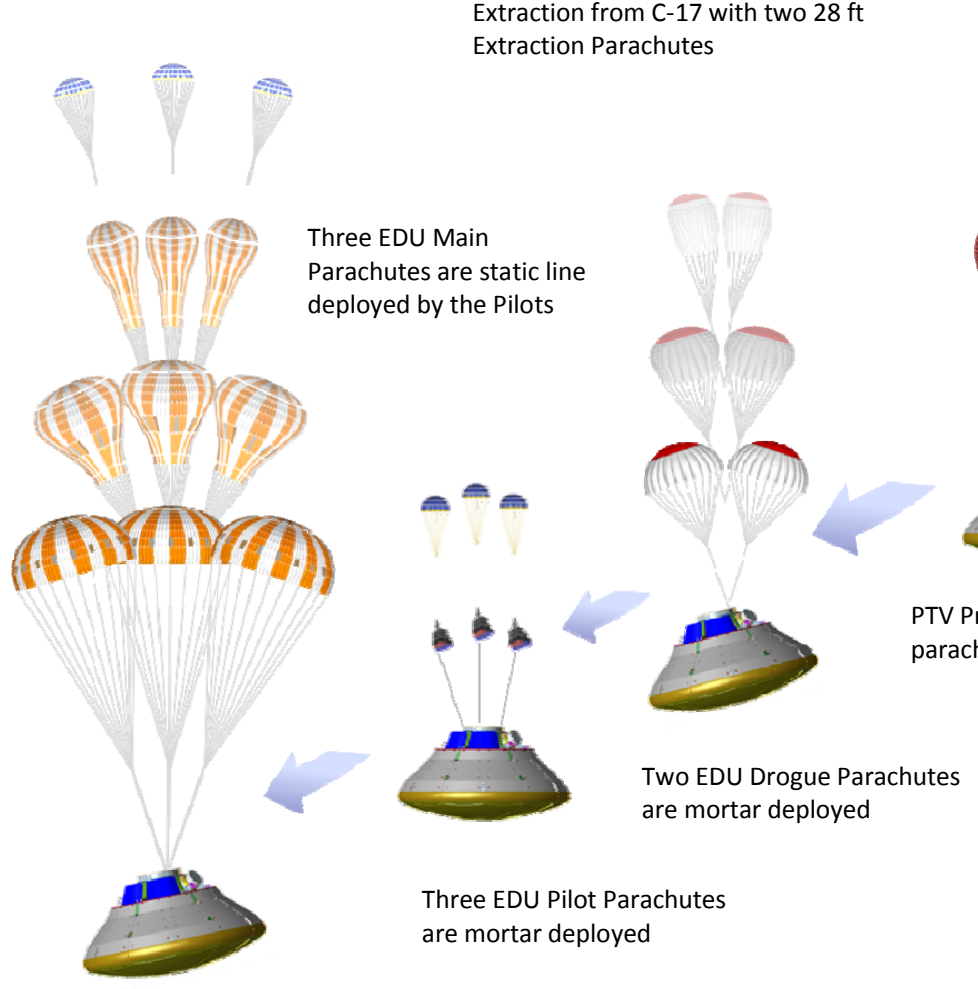

PTV/CPSS Separation \& PTV

Programmer Static Line Deployment
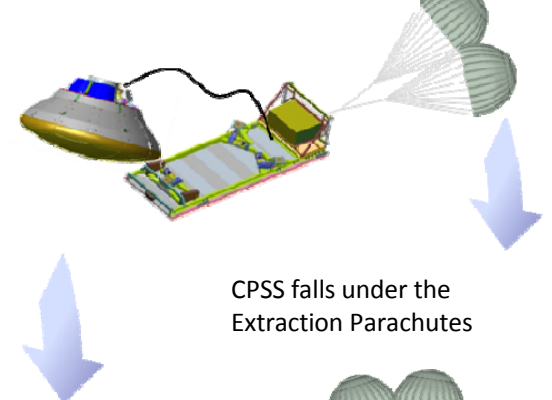

CPSS falls under the Extraction Parachutes
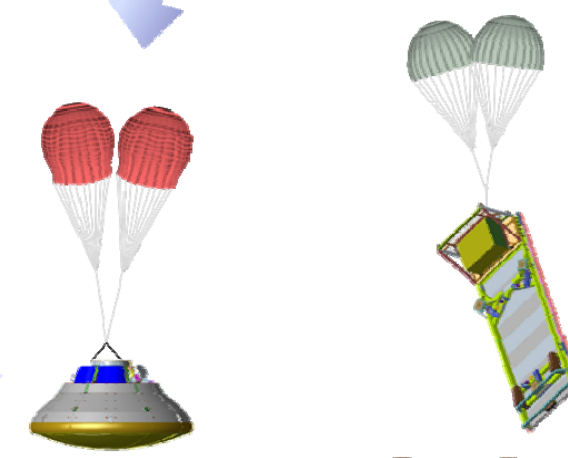

Parachutes are static line ployed by the Pilots

Figure 3. The PTV Test Sequence (including the CPSS recovery)

\section{Test Technique Evolution, and Hardware Improvements}

The CPAS Gen-I test vehicle sequencer systems relied exclusively on simple instrumentation and timed sequences to release the PTV following extraction. One positive result of the separation and subsequent free flight of the PTV during CDT-2 was this test demonstrated it was possible to get the PTV to a favorable condition for programmer inflation with this test architecture; heat shield into the wind with low total rates (figure 4). Following CDT-2 it was decided that, due to the uncontrollable and difficult to predict variations in LVAD extraction dynamics, a more sophisticated system would be required to provide a logic based separation capability, moving away from a purely time based test sequencer ${ }^{4}$. The sequencer would be upgraded to actively monitor the motion of the mated vehicle during extraction and release the restraint of the test vehicle such that the now free flying test vehicle would remain stable long enough to safely transition to the programmer. The inflated programmer would effectively 'catch' and orient the test vehicle without damaging it, and deliver it to the test point. This programmer damage requirement would be derived from how the programmer attaches to the test article and the range of attitudes the attach harness would pass through. The challenge, as was the case prior to CDT-2, would be developing the ability to model the test vehicle release and free flight, and have confidence the new approach would be repeatable in delivering the clean separation and programmer deployment condition observed on CDT-2. Several basic elements of the CDT-2 design were retained, most notably the interface of the PTV to the CPSS and the delayed load transfer during the LVAD extraction of the mated vehicle. 
A series of three LVAD platform tests were conducted following the completion of the CDT-2 investigation with a delayed load transfer to assess avionics hardware and software options that would eventually support an optimized test vehicle release algorithm. These tests did not involve the release of any test article from the platform following extraction from the aircraft. The goal of these tests was to support the development of models that would accurately reconstruct the extraction and under-rotation of the delayed load transfer LVAD platform, and in turn be used to predict the dynamics. In addition, these tests were used and to flight test potential avionics upgrades that would be used in the future. As a result of the TSE testing, a National Instruments c-RIO controller model 9025 was selected as the flight computer that would control the release of the test vehicle from the platform. The c-RIO is used to record data and send commands, both logic based and time based, to the firing relays. Crossbow NAV440 IMUs were selected to be the attitude and rate sensor package that would be used to optimize the test vehicle release. NovAtel SPAN- SE GPS/IMU units record detailed vehicle positional and vehicle orientation data during the test, however this data is processed after the test and is not used to make real time test sequencing decisions. The NovAtel is used to reconstruct the test vehicle and parachute system performance, providing significantly improved accuracy over the Gen-1 instrumentation suite used for this purpose.

\section{Review of CDT-2 Findings}

Reference 1 goes into a detailed description of the CDT-2 architecture, and the findings of the team that investigated the test failure. The root causes and contributing factors are repeated here as they defined the boundaries of how the team would go about designing the update to the test technique. Two root causes of the test failure, both involving inadequate prediction of wake effects, were identified:

1) The test design did not consider the effects of large pitch motions wrapping up the programmer and stabilization parachute risers which caused a decrease in the trailing distance.

2) The test design did not consider the wake deficit contribution of the stabilization parachutes on the programmer parachute performance.

The investigation team also identified three contributing factors:

1) Extraction and separation pre-test models were not robust. The resulting large dispersions of the PTV initial conditions resulted in addition of stabilization parachutes and the initial attach point on the side of the PTV.

2) Late completion of the PTV/CPSS separation Operations Concept resulted in PTV structural limitations which resulted in the requirement to reef the programmer parachute.

3) The programmer reefing configuration contributed to the parachute collapse and failure to re-inflate.

\section{Boiler Plate by way of Lawn Dart}

It is difficult to overstate the importance of the fact that the CDT-2 PTV/CPSS separation dynamics resulted in optimal PTV conditions at programmer deployment. As the project proceeded towards attempting to test a PTV again, decisions were always made with the knowledge that it was possible to achieve the desired heat shield forward orientation and low angular rates at programmer deploy (figure 4).

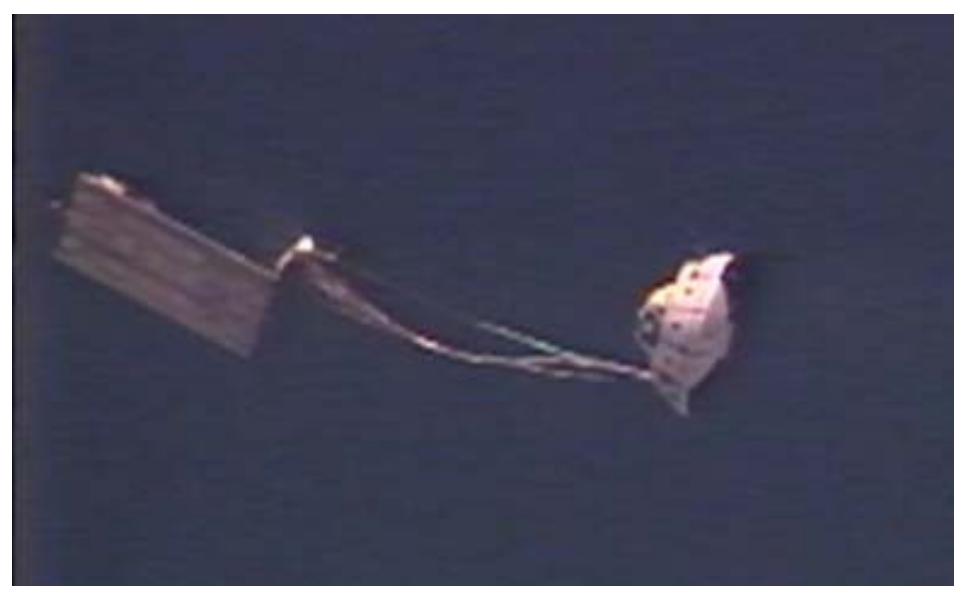

Figure 4. The CDT-2 PTV Attitude at Programmer Deployment 
The project decided that developing and executing the PCDTV release technique would precede the PTV testing. This decision was based on the fact that the PCDTV (by virtue of being a missile with fins and having large margin on static aerodynamic stability) would be less complicated to test with respect to modeling and identifying the optimum release conditions. The experience of modeling the PCDTV release would in turn drive down the risk of accurately predicting the optimum release point for the PTV which, due to the capsule aerodynamics and mass properties, is far less stable during free-flight and was expected to be more challenging to safely transition to the programmer phase of the test.

Similar to the PTV release architecture, the PCDTV test technique would use a delayed load transfer following the LVAD extraction, and would add a new element in the sequence. Following extraction, but prior to releasing the PCDTV, the Extraction Force Transfer Coupling (EFTC) would be released allowing the extraction parachute to reposition and remain attached to the MDS, now through the 'underside' of the MDS (figure 5). This reposition event would impart additional force and rotational rates, which if performed at the optimum time, would 'pitch' the PCDTV off of and clear of the platform. The concept for the new (intermediate reposition) element of the test technique came from an architecture that was being implemented by another program, the NASA Ares Jumbo Drop Test Vehicle (JDTV) ${ }^{5}$. The Ares test technique included a midair separation of the JDTV from its extraction platform. The CPAS test team adopted the JDTV test technique and adapted it to meet the PCDTV requirements. Although many modeling parameters had to be modified in the evolution from JDTV to PCDTV, the basic model was similar with a dart shape restrained in a saddle type structure.
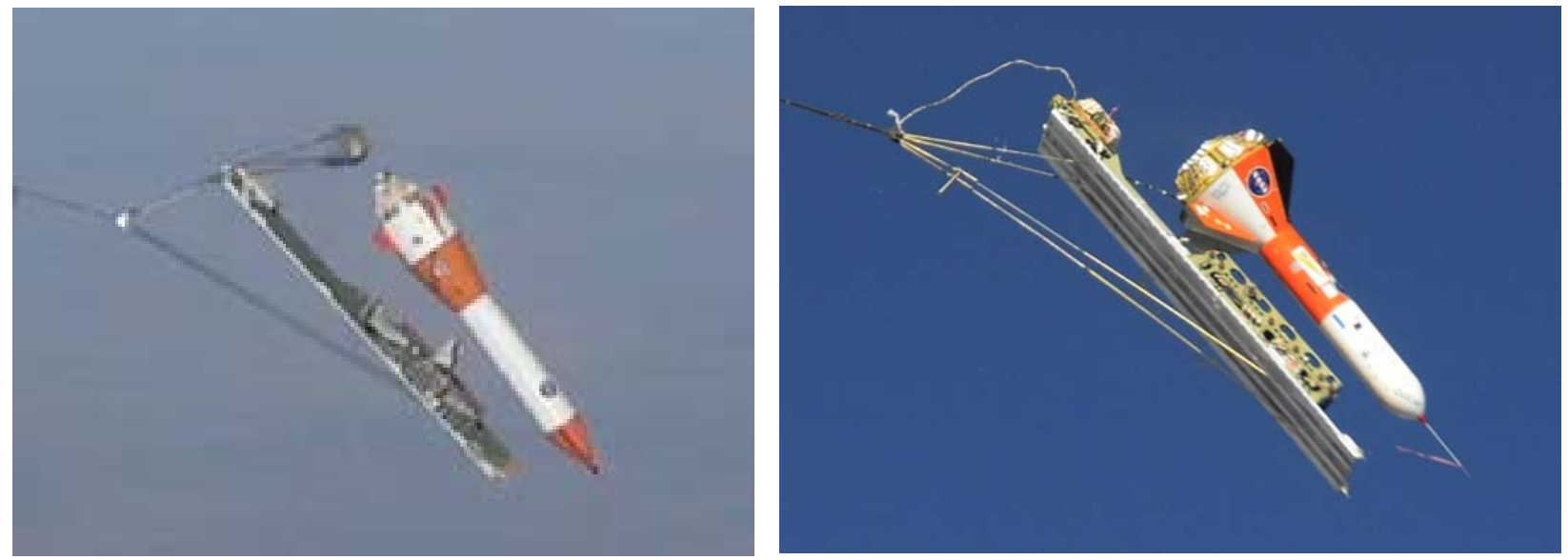

Figure 5. Release of the Ares JDTV (left) and the CPAS PCDTV (right) (immediately following intermediate reposition and release)

The NASA Ares Project also developed an ADAMS model to predict the JDTV extraction and midair separation. The ADAMS model combined physical contact effects related to how the test article was restrained to the platform, as well as the aerodynamic effects of the test article and the platform. These aerodynamic databases included the mated vehicle, the individual bodies free flying, and an increment for when the test vehicle was in close proximity to the platform following separation. CPAS funded a set of wind tunnel tests at the Texas A\&M Low Speed Wind Tunnel to collect this data for the CPAS configuration, and then supplemented the database with Computation Fluid Dynamics (CFD) solutions. The aerodynamic databases, used to predict the separation dynamics, were developed by the Orion Capsule Aerodynamics Project (CAP).

The CDT-2 version of the PTV/CPSS separation model did not include a physical contact model to predict the effect of the CPSS on the PTV separation initial conditions, rather the model attempted to predict the separation dynamics using aerodynamic parameters of the CPSS and PTV alone. Attempts to reconstruct the CDT-2 separation dynamics were unsuccessful until a mated vehicle contact model was included in the separation simulation. This led the simulation team to conclude a contact model was mandatory to have confidence in the predicted separation dynamics of the test vehicle. 


\section{Test Article Separation Logic and Constraints}

Two basic requirements were identified which generate the mated vehicle optimum release attitude and rate; 1) Prevent re-contact of the PCDTV with the MDS during initial separation, and 2) Avoid programmer riser contact with the released test vehicle throughout the programmer phase. The analysis required to identify the optimum release conditions (in terms of pitch and pitch rate) was a combination of several different models and simulations. The Decelerator Systems Simulation Application (DSSA ${ }^{6}$, a simplified version of DSS ${ }^{7}$ hosted in Excel) was used to model the combined platform/test vehicle from extraction parachute inflation all the way through PCDTV release from the platform. This included models for sliding out of the aircraft, tipping off the end of the ramp, and reposition from the Extraction Force Transfer Coupling to an intermediate re-position (holding the forward lower surface of the platform and the aft/trailing edge in the vicinity of the Load Transfer Coupling, as shown in figure 5). At this point in the trajectory the Decelerator Systems Simulation takes a hand off state vector for the PCDTV and models the trajectory of the test vehicle all the way through the remainder of the test. This includes models for the static line deployment of the programmer parachute, release of the programmer parachute and mortar deployment of the CPAS drogue parachutes, release of the drogue parachutes and mortar deployment of the CPAS pilot parachutes (which in turn deploy the main parachutes).

To avoid re-contact between the PCDTV and MDS, a geometric assessment determined that a clean separation would require a platform angle of attack $\alpha<-35^{\circ}$ at PCDTV release (see figure 6). For the PCDTV the programmer harness re-contact limit (in terms of alpha) was identified as $|\alpha|<90^{\circ}$ throughout the programmer phase of the trajectory. Due to the geometry of the structural attach points (through the top of the forward bay tunnel), this would keep the attach harness above the forward bay at all times. The tumbling requirement, defined as $|\alpha|<170^{\circ}$ throughout the programmer phase of the trajectory, was enveloped by the harness re-contact limit. Roll and roll rate were not considered to be factors in separation success, since the mated system would roll together, not creating any differential roll rates between the PCDTV and MDS, effectively the separation was treated as a pitch-plane solution. These factors set the success criteria for a parametric analysis of separation logic. End-to-end simulations using ADAMS to model the extraction through separation, and DSS to model the programmer phase of the test were run, varying the PCDTV release logic (mated pitch and pitch rate) until the optimum pitch and pitch rate conditions were identified, based on the success criteria. Once the optimized release logic was selected, Monte Carlos analyses were used to define a time window for the logic to execute to avoid a premature separation or a failure to separate if the logic conditions were not met. The time window had to open after the reposition event occurred, and close before the mated system damped out too much for a good separation.

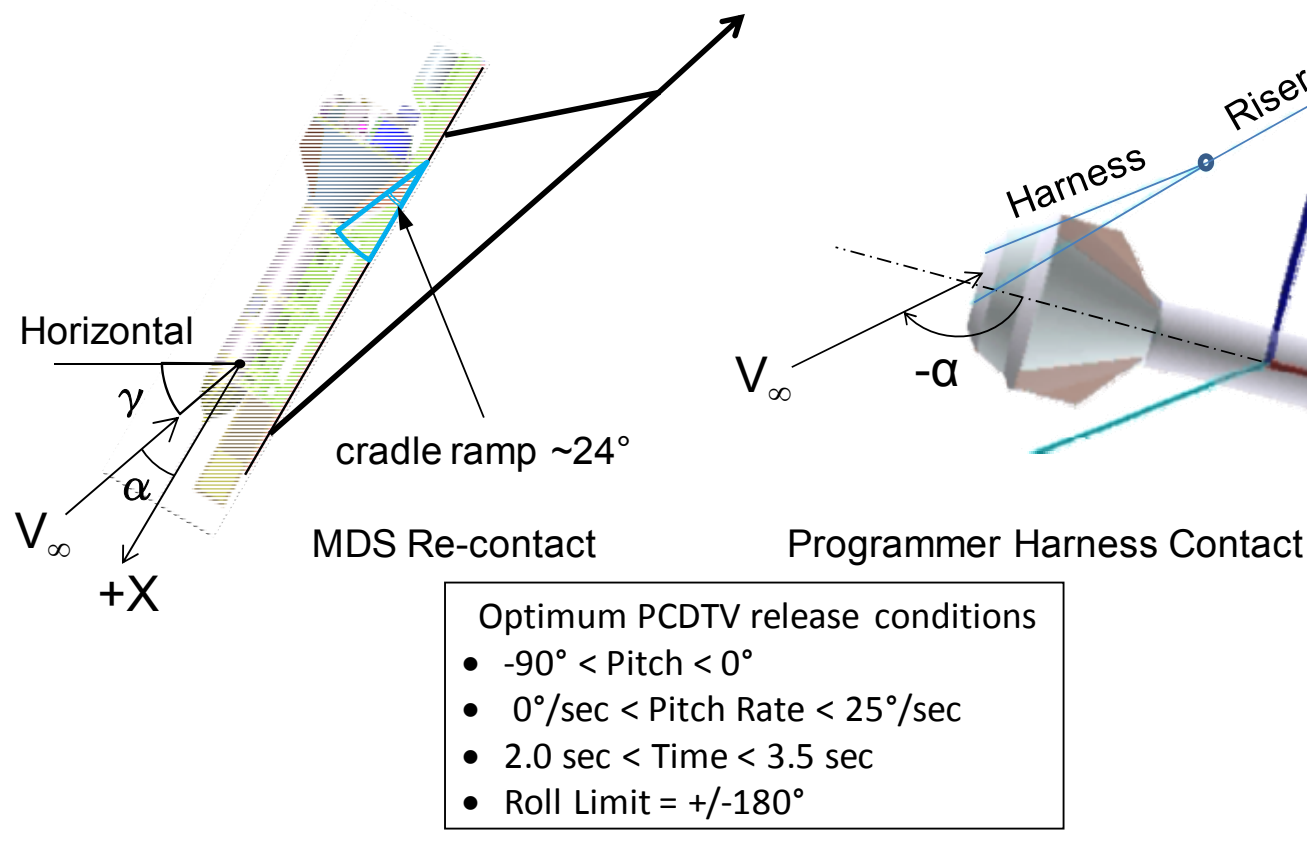

Figure 6. PCDTV Coordinate Systems and the Constraints for Release

Several goals were identified, not as requirements, but rather as criteria that would be optimized after identifying the release window. One such goal was to separate the PCDTV from the MDS as soon as possible following 
extraction and reposition. This was in order to try and preserve as much altitude as possible (and get on test point), and in part due to the lack of confidence in predicting the motion of the mated vehicle (effectively a large flat plate). There were other benefits associated with early separation such as the 'bucket' in dynamic pressure that occurs immediately following an LVAD extraction, and the associated lower programmer inflation loads, that were thought to be a benefit to this approach. Another goal was to minimize oscillations of the PCDTV, with the downstream desire to get on test point quickly and not have large residual PCDTV rates at programmer release. The project chose to use CPAS parachutes (initially the drogues) as programmer parachutes. This is relevant to the modeling in that it established the separation distance the PCDTV would need to achieve in order to deploy the programmer parachute, which in turn would arrest or 'catch' the test vehicle. The benefit was to gather more data on CPAS parachute performance.

The test vehicle release window open and close times would be based on simulations, and attempt to mitigate the risk of a bad release without over constraining the release logic. The window 'open' would protect against a premature/too early separation, and re-contact with the MDS. The window 'close' would protect against an avionics failure, which could result in the release logic conditions never being met.

The end to end simulation was used to explore and characterize the release window. First, a sweep of times for the intermediate reposition (EFTC release) was performed to identify the MDS reposition time that created the largest window for PCDTV release. The MDS reposition event would not be triggered by the avionics, rather it was a hard coded delay time from ramp clear. The 'real time' decision (using the onboard sensors) for releasing the PCDTV would open after the MDS reposition event. The decision to hard code the intermediate reposition time was made in order to simplify the algorithm, and with anticipation that the general physics (periodic motion) observed in the JDTV test architecture would be repeatable for the lighter PCDTV configuration.

The optimum time to release the PCDTV from the mated vehicle was predicted to take place around the minimum in alpha with an ascending pitch rate. This is plotted graphically in figure 7 . The left hand plot is mated vehicle angle of attack vs. time from ramp clear, while the right hand plot is mated vehicle pitch rate vs. time from ramp clear. The red line (in either plot) is what was considered the nominal prediction. The remainder of the predicted trajectories are as a result of varying parameters within the simulation to account for variations in: the aircraft extraction (altitude, velocity, ramp angle, extraction chute performance), platform and test vehicle mass properties, and aerodynamic models. The data are plotted vs. time to provide a frame of reference that represents how the sequencer would interrogate the data (with respect to release window open/close). The yellow box in each plot represents the open and close times for the optimized release algorithm. The datum of time from ramp clear is physically tied to a series of pins that are pulled (throwing switches) as the mated vehicle physically clears the trailing edge of the aircraft ramp.
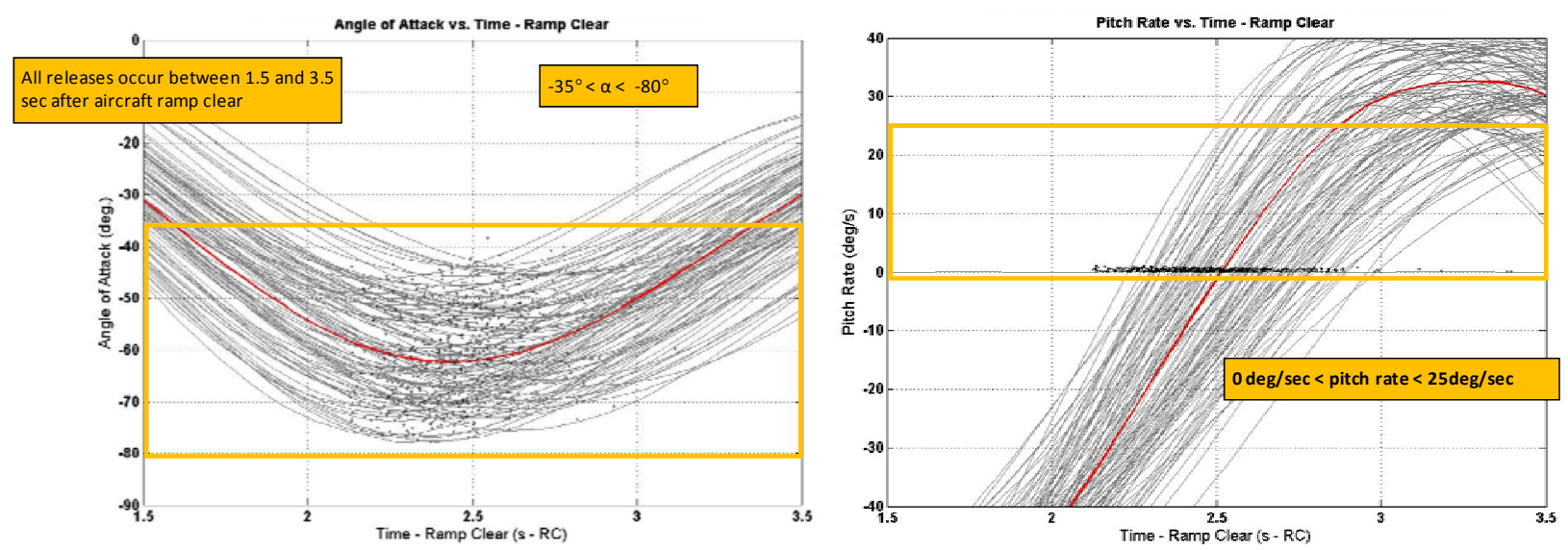

Figure 7. Monte Carlo Predictions of Mated Vehicle Dynamics and the Release Logic Window

The first two tests (CDT 3-1, and CDT 3-2) released the PCDTV on time rather than logic because the natural period during the under-rotation of the mated vehicle was longer than predicted, primarily due to aircraft pitch during extraction. This longer period resulted in the time to achieve the optimum conditions taking longer than predicted. On both tests the separation was good enough to result in no re-contact between the test vehicle and either the MDS or programmer attach harness following release. The programmer parachute successfully righted the test vehicle without total alpha becoming less than 90 degrees, and there was no harness re-contact with the 
forward bay throughout the programmer phase. Several changes were made prior to the third test. Aircraft pitch predictions were updated in the extraction model and the time window was extended to allow for other un-modeled factors affecting mated system dynamics. Additionally, two extraction parachutes were used (instead of the single extraction parachute used for the first two tests). Finally, the release window close time was extended to 3.5 seconds after aircraft ramp clear. The separation was successfully triggered based on pitch and pitch rate logic for the third PCDTV test.

\section{The PTV Separation Solution}

The evolution of the separation model from PCDTV to PTV was a major revision to not only the models but how to interpret the simulation results. The aerodynamic parameters were updated to reflect a capsule shape, and the contact model was updated to reflect the three point attach and adjacent skid plates. The ADAMS PTV/CPSS (mated vehicle) extraction simulation tool models the mated vehicle dynamics all the way through release of the PTV. This includes the following phases of the test; extraction chute inflation, mated vehicle slide-out on the aircraft ramp, tipping as the mated vehicle clears the ramp, and contact between the PTV and CPSS during the PTV separation. The DSS tool initializes the PTV with a handoff state from the ADAMS tool. The DSS tool models the dynamics of the PTV just after separation from the CPSS all the way to landing. The DSS simulation includes the following phases of the test; the static line deployment of the programmer, coupled PTV and programmer trajectory and dynamics, programmer release, drogue mortar fire, drogue inflation/disreef, coupled PTV and drogue trajectory and dynamics, drogue release, main pilot mortar fire, main bag extraction, main inflation/disreef, and the coupled PTV and main trajectory and dynamics all the way to landing. As with the PCDTV analysis, all the simulations model the parachute as a rigid 6-DOF body attached to the payload by an elastic suspension system (riser and/or harness). The PTV is modeled as a separate 6-DOF body. Each body has its own independent aerodynamic force and moment database from legacy models, wind tunnel testing, and CFD analysis. This includes an increment to the PTV aero due to proximity to the CPSS as they separate.

The ADAMS separation model was validated incrementally. The JDTV version was validated by reconstructing JDTV airdrop testing. The PCDTV version was validated by similarity to JDTV for the first test and by reconstructing subsequent the PCDTV tests as they were performed (CDT-3-1, CDT-3-2, CDT-3-4, and CDT-36). The PTV version was validated by reconstruction of CDT-2, using the limited data available due to loss of the test vehicle during the test. Post test reconstructions of EDU PTV tests (CDT 3-3, CDT 3-5, CDT 3-7) have continued to validate and improve the ADAMS model. The DSS simulation has been used and constantly verified throughout the CPAS project by reconstructing various types of air drop tests that have been conducted.

From preliminary simulations of the PTV release, it became apparent that the margin between a successful release and transition to the programmer parachute was significantly smaller than with the PCDTV; the PTV would tend to flip apex forward significantly quicker than the PCDTV. This was in part due to the phasing of pitch and pitch rate, and in part due to the aerodynamic and mass properties of the PTV; This resulted in the window to deploy the programmer to be smaller. The optimum release condition was again identified in pitch and pitch rate space, shown in figure 8 . The simulation time history traces in figure 8 are read from platform clear (positive pitch angle with near zero rate), counterclockwise through the platform under rotation oscillation (due to the delayed load transfer of the extraction parachute), into the PTV release window identified by the red box. The initial positive pitch angle as the mated vehicle clears the aircraft ramp is due to the aircraft pitching nose up as the mated vehicle moves aft and changes the center of gravity of the aircraft. Defined as time from ramp clear, the Monte Carlo simulation runs predicted the optimum release conditions would be achieved in 1.7 to 2.3 seconds. 


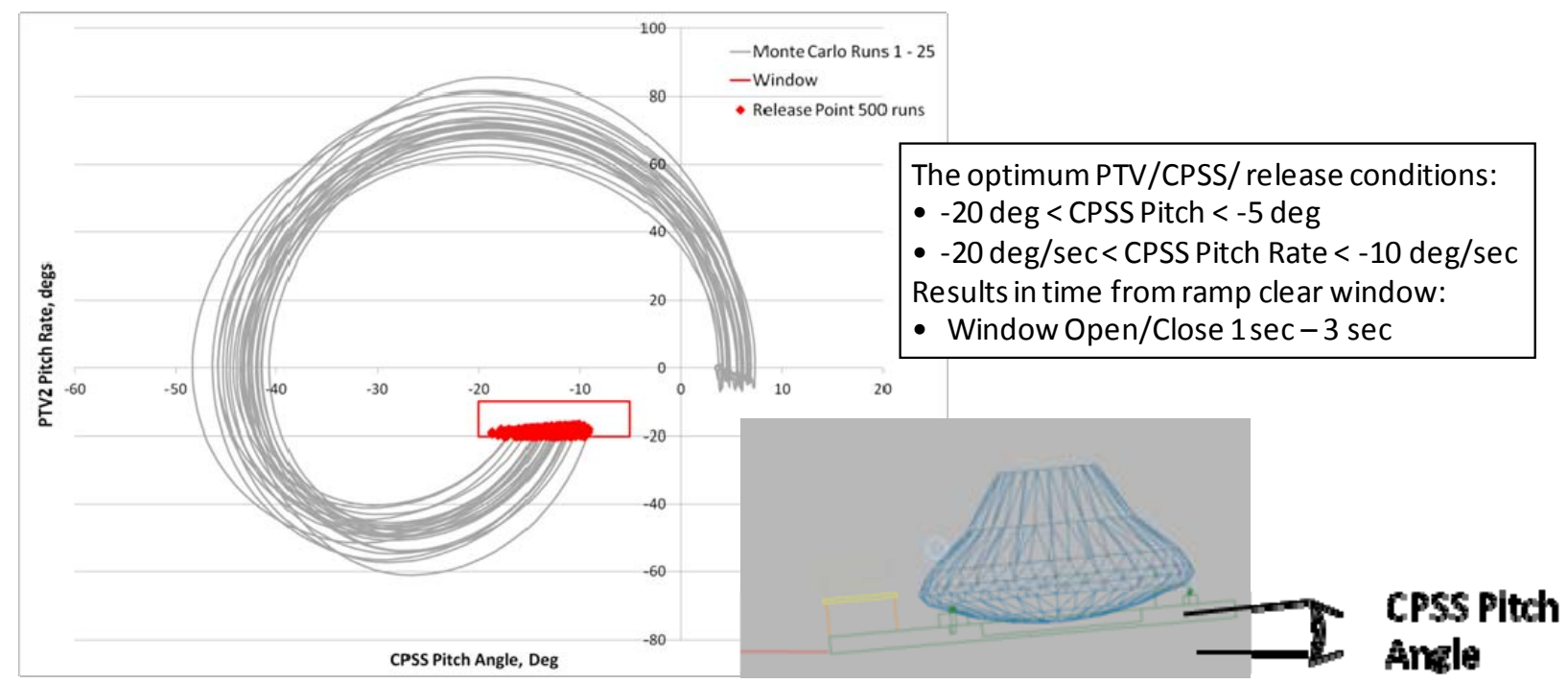

Figure 8. ADAMS Simulation: Ramp Clear to PTV Release Window

As with the PCDTV, the pitch angle at release controls the risk of the PTV re-contacting the CPSS, while the pitch rate dominates the risk of the PTV tumbling (either prior to programmer inflation, or afterwards) allowing the programmer harness to come in contact with the PTV. Similar to the PCDTV approach, the window open and close protects against releasing on time, increasing the likelihood of releasing on optimized pitch and pitch rate. Potential causes identified for releasing on time were related to a failure of the avionics system (a bad Inertial Measurement Unit for instance), a rigging error resulting in the ramp clear flag not being triggered properly, or an over constrained window due to poor trajectory modeling (either opening too late, or closing too soon). To establish the open and close times for the PTV release window, the end to end simulation (ADAMS handing off to DSS) had to be run repeatedly, forcing the release to take place on time alone. The ADAMS simulation was run in a Monte Carlo fashion with release forced to occur in 1/10 second increments moving away from the optimum release time in either direction. The results were tabulated to understand the likelihood that one of the constraints (PTV contact with the CPSS, or programmer harness contact with the PTV) would be violated. Table 1 summarizes the percentage of successful cases (varying either the window open or close value).

\begin{tabular}{|c|c|c|c|c|c|c|c|c|c|c|c|c|c|c|c|c|c|c|}
\hline $\begin{array}{c}\text { *02/16/12 } \\
\text { ADAMS/DSs } \\
\text { Iteration 2 }\end{array}$ & \multicolumn{9}{|c|}{ WINDOW OPEN } & \multicolumn{1}{c|}{ WINDOW CLOSE } \\
\hline $\begin{array}{c}\text { Time } \\
\text { Slices } \\
\text { (sec-RC) }\end{array}$ & $\mathbf{1 . 0}$ & $\mathbf{1 . 1}$ & $\mathbf{1 . 2}$ & $\mathbf{1 . 3}$ & $\mathbf{1 . 4}$ & $\mathbf{1 . 5}$ & $\mathbf{1 . 6}$ & $\mathbf{1 . 8}$ & $\mathbf{2 . 1}$ & $\mathbf{2 . 2}$ & $\mathbf{2 . 3}$ & $\mathbf{2 . 4}$ & $\mathbf{2 . 6}$ & $\mathbf{2 . 8}$ & $\mathbf{3 . 0}$ & $\mathbf{3 . 5}$ & $\mathbf{3 . 8}$ & $\mathbf{4 . 0}$ \\
\hline $\boldsymbol{\alpha}>\mathbf{3 5 0}$ & 99 & 95 & 97 & 75 & 43 & 20 & 4 & 1 & 35 & 56 & 76 & 90 & 95 & 99 & 99 & 73 & 45 & 22 \\
\hline $\boldsymbol{\alpha}>\mathbf{2 7 0}^{\circ}$ & 99 & 96 & 98 & 79 & 47 & 23 & 5 & 1 & 38 & 60 & 78 & 91 & 95 & 99 & 99 & 75 & 46 & 25 \\
\hline $\boldsymbol{\alpha}<\mathbf{9 0}^{\circ}$ & 98 & 95 & 97 & 76 & 44 & 21 & 6 & 3 & 36 & 58 & 77 & 90 & 99 & 99 & 99 & 73 & 45 & 24 \\
\hline
\end{tabular}

All times are relative to Ramp Clear (sec-RC); Initialized with dispersed ADAMS ICs

Table 1. Risk Trade for Determining Release Window Open and Close

Figure 9 tracks the trajectory of the PTV in the pitch rate vs. alpha space from ramp clear through PTV programmer deploy (plotted in the PTV reference frame). The yellow points in this plot correspond to the red points in figure 8 , associated with the optimum release conditions. The range of attitude and rate at programmer deploy (the red points in figure 9) influences the initial free flight of the PTV, which ends when the programmer inflates and dominates the dynamics of the PTV. 


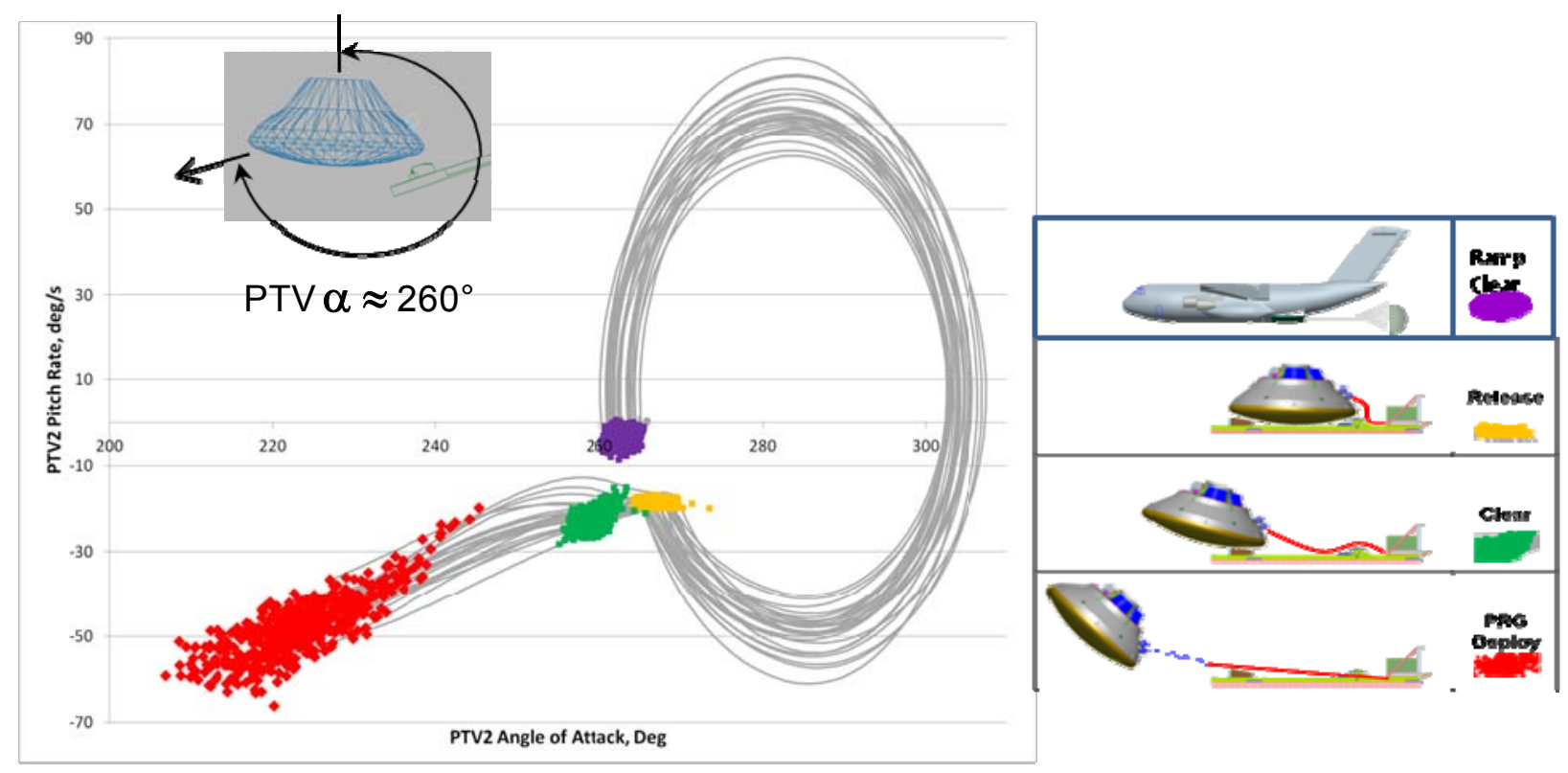

Figure 9. Ramp Clear through Programmer Deploy in PTV Reference Frame

The results from several simulations, from extraction through programmer stabilization of the PTV, are displayed in two different ways in figure 10. In both plots, the ADAMS simulation is the dashed line, and the DSS simulation is the solid line. The left hand plot is a time history of the PTV angle of attack. The effectiveness of the programmer parachute (inflation at roughly 5 seconds) to damp the PTV pitch motion is clear. This is primarily due to the programmer having a multipoint attach harness that passes through the forward bay tunnel (well above the center of gravity of the PTV). From this plot it is also apparent that when the programmer inflates with a large PTV alpha the rates do not appear to damp completely prior to the desired programmer release time. The right hand plot is similar, only this time the trajectory is plotted as PTV pitch rate vs. angle of attack, with the PTV release implementing the optimized release window (red box from Figure 8). The effect of limiting the rate of the mated vehicle at PTV release is to eliminate the runs that attempted to go apex forward (the top trace on the left hand plot) as well as the ones that tended to not damp in the programmer phase (mated vehicle rates of greater than $30 \mathrm{deg} / \mathrm{sec}$ on the left hand plot).

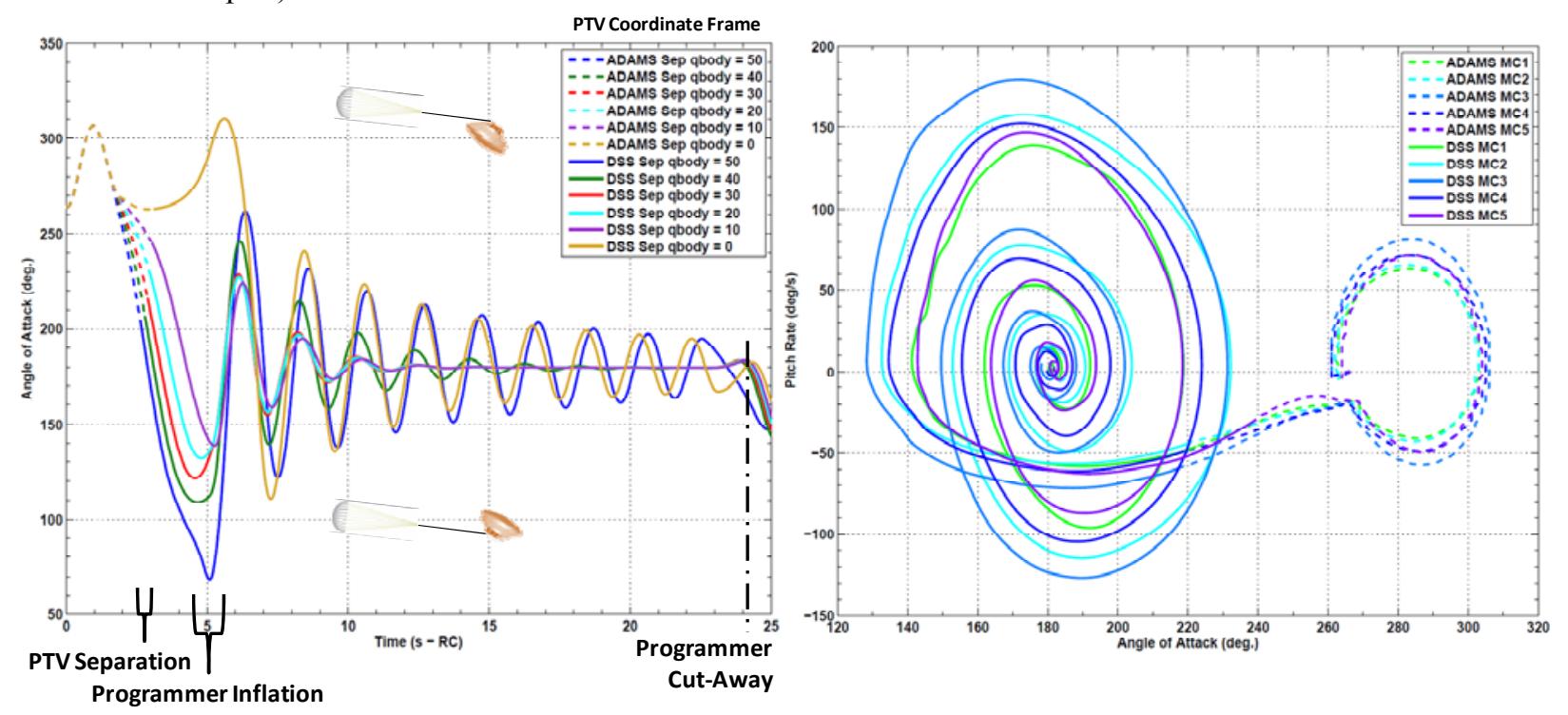

Figure 10. Ramp Clear Through Programmer Terminal Trajectory 
Figure 11 is a series of still images taken during extraction and release of the PTV during CDT-3-5 (the second test of the PTV using the optimized release technique). The under rotation prior to release, and the programmer parachutes being static line deployed are clearly visible, with the fully inflated programmer parachutes visible in the last image. The CPAS project has successfully executed this technique all three times it has been attempted.

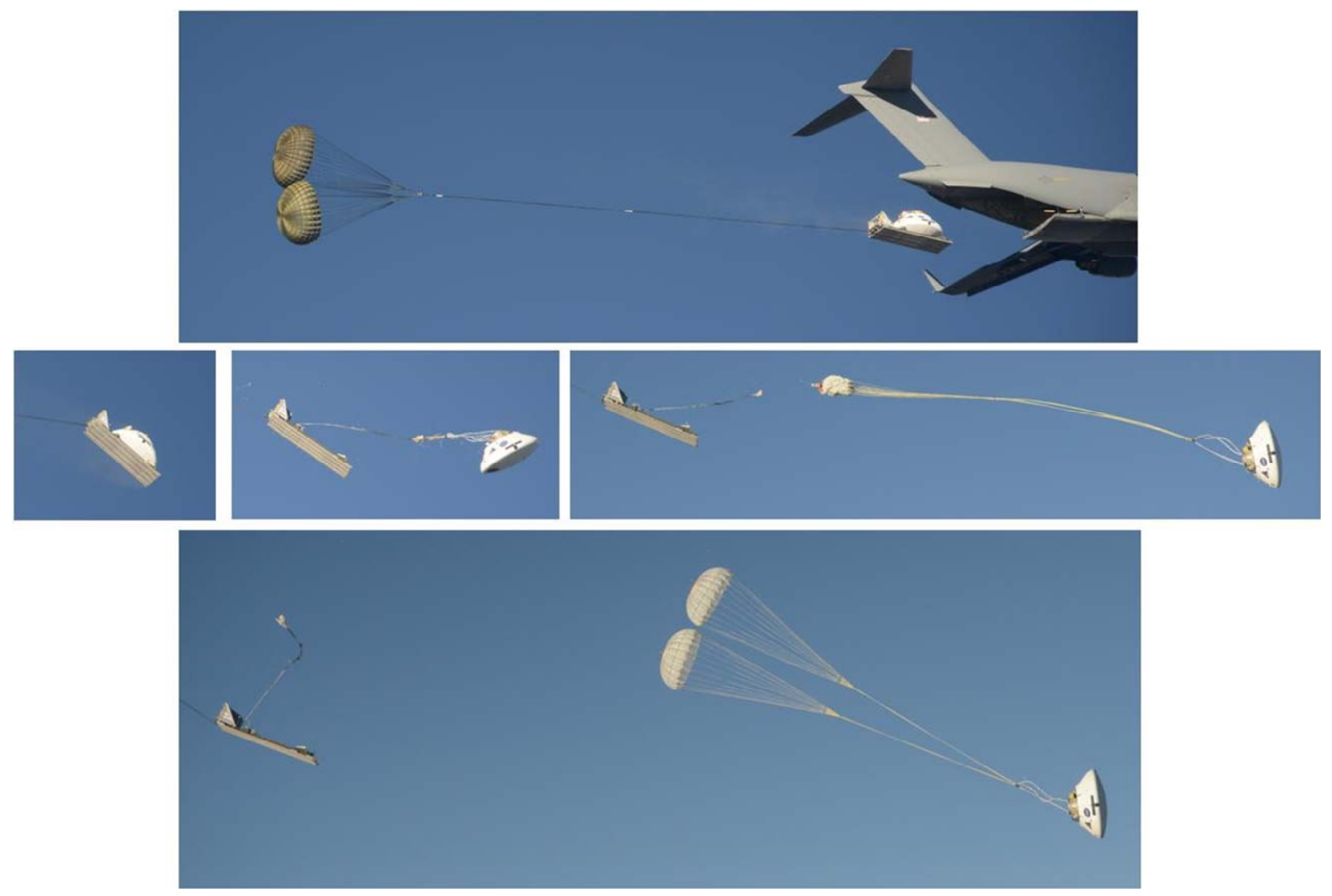

Figure 11. CDT-3-5 Extraction and Release Sequence

\section{Conclusion}

The approach and simulation tools described have provided a means for understanding how to safely get either the PCDTV or the PTV on test point starting with an LVAD test technique. The avionics chosen have demonstrated to be robust and reliable, providing a means to monitor the extraction system performance and allow the sequencer to make a 'real time' decision on releasing the test vehicle. Monte Carlo runs were necessary to identify the optimum release conditions. The solution is test vehicle dependent in terms of the databases used to predict the release window. A release window, as a function of time, was established in order to mitigate the risk of an avionics failure resulting in releasing at an inopportune condition. As of January 2013, the CPAS project has successfully executed four PCDTV tests and three PTV tests. All three of the PTV tests have released on optimized conditions (and neither time based on either release window limit). The approach discussed has allowed the analysis and test teams to investigate and begin to implement smaller programmer parachutes in order to achieve more demanding test conditions. This approach, modified with new re-contact constraints, will be used in designing the PTV tests that involve the jettison of the Forward Bay Cover on test condition. The CPAS project also anticipates using this approach to the Qualification testing of the design that emerges from the Critical Design Review, in support of human rating the Orion Spacecraft.

\section{Acknowledgments}




\section{References}

\footnotetext{
${ }^{1}$ Machin, R.A. and Evans, C.T., "Cluster Development Test 2 an Assessment of a Failed Test," May 2009, 20th AIAA Aerodynamic Decelerator Systems Technology Conference, Seattle, Washington.

${ }^{2}$ Taylor, A.P., Machin, R.A., and Royall, P., "Developing the Parachute System for NASA's Orion - An Overview at Inception," May 2007, 19th AIAA Aerodynamic Decelerator Systems Conference, Williamsburg, Virginia.

${ }^{3}$ Ray, E., "Challenges of CPAS Flight Testing," May 2011, 21st AIAA Aerodynamic Decelerator Systems Technology Conference, Dublin, Ireland.

${ }^{4}$ Moore, J.W., "Development of a Smart Release Algorithm for Mid-Air Separation of Parachute Test Articles," May 2011, 21st AIAA Aerodynamic Decelerator Systems Technology Conference, Dublin, Ireland.

${ }^{5}$ King R., Hengel J., and Wolf D., "Ares Decelerator System Heavy Air Drop Test Program," May 2011, 21st AIAA Aerodynamic Decelerator Systems Technology Conference, Dublin, Ireland.

${ }^{6}$ Cuthbert, P.A. and Conley, G.L., "A Desktop Application to Simulate Cargo Drop Tests," May 2005, 18th AIAA Aerodynamic Decelerator Systems Technology Conference, Munich, Germany.

${ }^{7}$ Cuthbert, P.A. and Desabrais, K.J. "Validation of a Cargo Airdrop Software Simulator," May 2003, 17th AIAA Aerodynamic Decelerator Systems Technology Conference, Monterey, California.
} 